\title{
Avanços nos materiais e no tratamento endovascular de oclusões arteriais crônicas totais: um relato de caso
}

\author{
Advances in materials and endovascular treatment of chronic total arterial occlusions: a case report
}

Daniel Queiroz Neves', Jorge Ribeiro da Cunha Junior², Márcio Cerbazzi Tavares Cardoso3, Mauro Henrique de Lima33, Gustavo Petorossi Solano ${ }^{4}$, Celso Luis Muhlethaler Chouin ${ }^{4}$, Sérgio Lopes de Azevedo ${ }^{5}$, Paulo Eduardo Ocke Reis ${ }^{6}$

\begin{abstract}
Resumo
As oclusões arteriais crônicas totais com forte componente cálcico são ainda nos dias atuais, um fator muitas vezes limitante para o tratamento endovascular devido à dificuldade em transpor estas lesões com fios-guia e cateteres habitualmente utilizados. Revisamos a literatura e descrevemos um caso de tratamento endovascular de uma oclusão total de artéria ilíaca externa, onde o uso de novos materiais desenvolvidos especificamente para o tratamento deste tipo de lesão foi determinante para o sucesso do caso.
\end{abstract}

Palavras-chave: arteriopatias oclusivas; arteriosclerose obliterante; claudicação intermitente.

\begin{abstract}
Chronic arterial occlusions with great calcium component are usually a factor of limitation to endovascular treatment to the difficulty to transpose these lesions with guidewires and catheters commonly used. We reviewed the literature and described a case of endovascular treatment of a total occlusion of external iliac artery, where the use of new materials developed specifically to the treatment of such injuries was critical to the success of the case.
\end{abstract}

Keywords: arterial occlusive diseases; arteriosclerosis obliterans; intermittent claudication.

\section{Introdução}

A doença aterosclerótica obstrutiva crônica periférica ainda é, na prática clínica atual, um sério problema que reduz a qualidade de vida e incapacita nossos pacientes, cuja prevalência atualmente encontra-se em torno de $20 \%{ }^{1}$ e tende a aumentar com o envelhecimento da população. Agravando tal situação, temos a associação de outras comorbidades frequentes na população de idade mais avançada ${ }^{2-4}$. A técnica endovascular surgiu como uma alternativa viável e segura para o tratamento deste grupo de pacientes, firmando-se como uma das opções de primeira escolha no tratamento das lesões obstrutivas do eixo aorto-ilíaco em pacientes com risco cirúrgico elevado ou proibitivo à técnica convencional de revascularização. No entanto, esta técnica apresenta limitações no tratamento de lesões obstrutivas longas e calcificadas que conferem oclusão à luz arterial, frente ao índice de insucesso em transpor lesões deste tipo com os fios-guia disponíveis².

Este artigo visou demonstrar como as inovações na área de bioengenharia e o aprimoramento dos materiais destinados à cirurgia endovascular podem e vêm diminuindo as limitações da técnica minimamente invasiva, ajudando estes pacientes cuja possibilidade terapêutica convencional torna-se de elevado risco ou inapropriada devido às comorbidades e estado clínico.

Trabalho realizado no Hospital Universitário Antônio Pedro (HUAP) da Universidade Federal Fluminense (UFF) - Niterói (RJ), Brasil.

${ }^{1}$ Médico Residente (R2) do Serviço de Cirurgia Vascular do HUAP da UFF - Niterói (RJ), Brasil.

${ }^{2}$ Médico Residente (R1) do Serviço de Cirurgia Vascular do HUAP da UFF - Niterói (RJ), Brasil.

${ }^{3}$ Médico Pós-graduando (P2) do Serviço de Cirurgia Vascular do HUAP da UFF - Niterói (RJ), Brasil.

${ }^{4}$ Staff do Serviço de Cirurgia Vascular do HUAP da UFF - Niterói (RJ), Brasil.

${ }^{5}$ Enfermeiro do Serviço de Cirurgia Vascular do HUAP da UFF - Niterói (RJ), Brasil.

${ }^{6}$ Chefe do Serviço de Cirurgia Vascular do HUAP da UFF - Niterói (RJ), Brasil.

Conflito de interesse: nada a declarar.

Submetido em: 10.08.10. Aceito em: 29.02.12.

J Vasc Bras. 2012;11(3):226-231. 
Descrevemos aqui o caso de uma paciente tratada no Hospital Universitário Antônio Pedro, com lesão ilíaca TASC tipo $B^{3}$, estágio III de Fontaine ${ }^{4}$, grau II - categoria 4 de Rutherford ${ }^{5}$-, que se enquadra neste grupo de doentes de elevado risco, cujo tratamento eficaz seria dificultado ou até mesmo inviável, não fosse o uso de novos materiais inovadores com finalidades específicas que possibilitaram a transposição de uma lesão obstrutiva complexa que não fora vencida pelos meios tradicionais em tentativa prévia, durante o mesmo procedimento, sem o uso dos referidos materiais.

\section{Relato do caso}

C.L.E.S., 58 anos, parda, natural de Maricá (RJ), atendida no ambulatório de cirurgia vascular do Hospital Universitário Antônio Pedro em maio de 2010 queixando-se de dor na perna direita com piora progressiva há seis anos, principalmente em panturrilhas, intensificada à deambulação. A paciente referiu parestesia em ambos os pés, porém mais intensa à direita. Já em uso de cilostazol $100 \mathrm{mg}$ duas vezes ao dia e AAS $100 \mathrm{mg}$ uma vez ao dia há cerca de três anos com progressão dos sintomas até o aparecimento de dor em repouso há aproximadamente três meses. Não houve melhora da dor com analgésicos não opioides.

Ao exame físico não apresentou lesões tróficas, ausência de pulsos femoral, poplíteo e distais em membro inferior direito, estando os mesmos presentes no lado oposto. Apresentou palidez cutânea em região plantar direita e hiperemia reativa deste pé. Índice-tornozelo-braquial (ITB) 0,40 à direita e 0,80 , à esquerda.

História patológica pregressa: diabética em uso de insulina NPH já com retinopatia e microalbuminúria; hipertensa em uso de Losartan $50 \mathrm{mg}$ duas vezes ao dia, captopril $25 \mathrm{mg}$ duas vezes ao dia e carvedilol 0,025 mg uma vez ao dia; dislipidêmica em uso de sinvastatina $40 \mathrm{mg}$ uma vez ao dia. Ex-tabagista com carga tabágica de 25 maços/ano, abstinente há dois anos.

Foi indicada arteriografia para investigação e melhor definição de conduta quanto à provável oclusão arterial no leito ilíaco-femoral uma vez que não dispúnhamos à época de angiotomografia em nosso hospital.

$\mathrm{Na}$ arteriografia, observou-se oclusão de artéria ilíaca externa estendendo-se desde sua origem até a artéria femoral comum onde reabita por vasta rede de colaterais (Figuras 1 e 2). O leito arterial distal à oclusão apresentou doença aterosclerótica difusa, porém sem nenhuma estenose significativa e patência das três artérias distais até o pé.

A avaliação do risco cirúrgico mostrou-se elevada para realização de derivação aorto-femoral devido ao padrão de cardiopatia isquemia prévia no ECG e ao ecocardiograma.

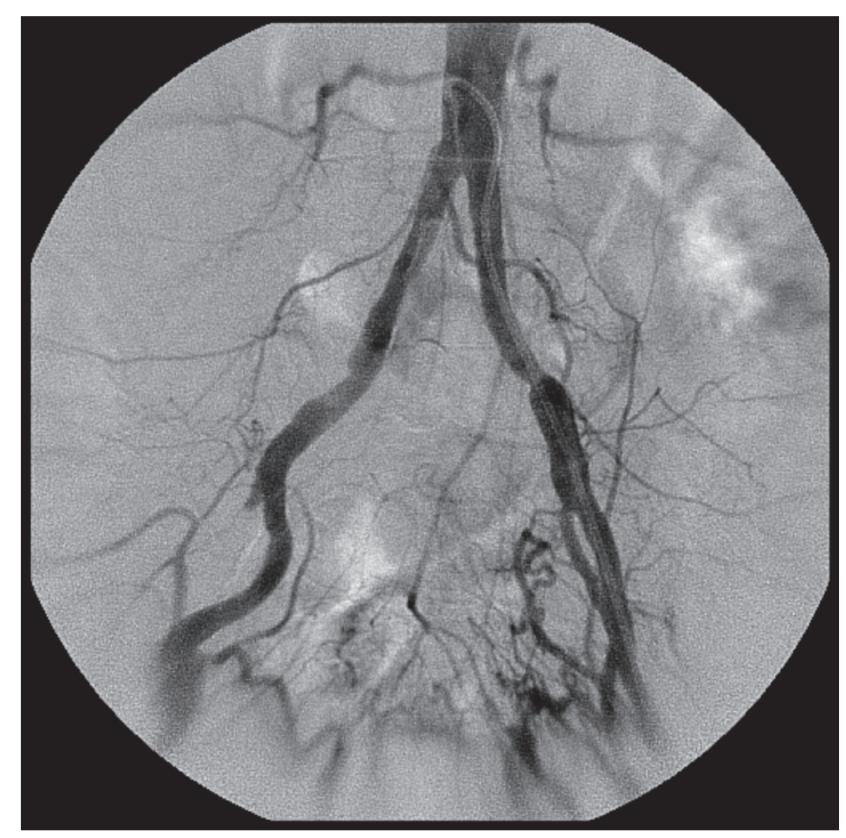

Figura 1. Arteriografia aorto-ilíaca.

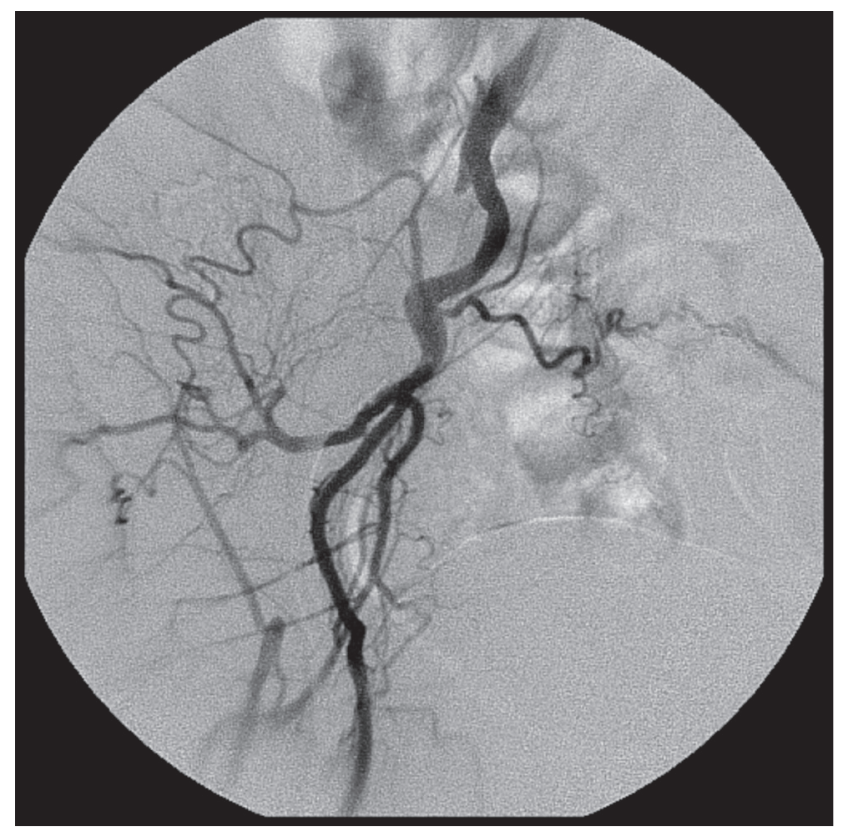

Figura 2. Oclusão total de artéria ilíaca externa.

Mediante os riscos, optou-se pela abordagem endovascular como primeira escolha de tratamento. Caso esta não fosse possível ou efetiva, optaria-se por uma derivação extra-anatômica fêmoro-femoral cruzada.

O procedimento de angioplastia transluminal percutânea proposto foi realizado no Serviço de Hemodinâmica do Hospital Universitário Antônio Pedro em 17 de junho de 2010, sob anestesia raquimedular. Durante o procedimento, foi realizado acesso por punção da artéria 
femoral esquerda com cruzamento da bifurcação das artérias ilíacas com cateter diagnóstico mamária, seletivando a artéria ilíaca comum direita. Foi realizada a introdução de bainha contralateral tipo Balkin 7F posicionando-a na emergência da artéria ilíaca externa após heparinização sistêmica com 2.500 unidades de heparina não fracionada. Ao tentar vencer a lesão com guia hidrofílico 0,035 ", não houve progressão do mesmo devido às características da placa ateromatosa de longa evolução, já com calcificação significativa (Figuras 3 e 4). Desta forma, utilizou-se o cateter Frontrunner ${ }^{\circledR}$ para transposição de lesões calcificadas sob microguia específico $3 \mathrm{~F}$, que possibilitou a transposição da lesão (Figura 5), porém à passagem do fio-guia hidrofílico 0,014 " através do microcateter, o mesmo mostrou-se subintimal após a transposição da oclusão. Assim sendo, utilizou-se um cateter de reentrada Outback ${ }^{\circledR}$ (Figura 6) para reintrodução do guia na luz arterial (Figura 7), o que possibilitou a angioplastia transluminal com balão de $3,5 \mathrm{~mm}$ (Figura 8) seguida de implante de stent de $8 \times 100 \mathrm{~mm}$ e balonamento pós-colocação do stent com balão de $6 \mathrm{~mm}$ para melhor acomodação do mesmo (Figura 9).

A arteriografia de controle ainda apresentava estenose residual de cerca de $50 \%$ logo após o término do stent, o que justificou outra angioplastia por balão nesta região, desta vez bem sucedida e com aspecto arteriográfico satisfatório (Figura 10). Prosseguiu-se com a retirada do sistema coaxial sob visão direta e colocação de selante hemostático de colágeno $8 \mathrm{~F}$ no sítio de punção, seguido de curativo compressivo e aquecimento dos membros inferiores.

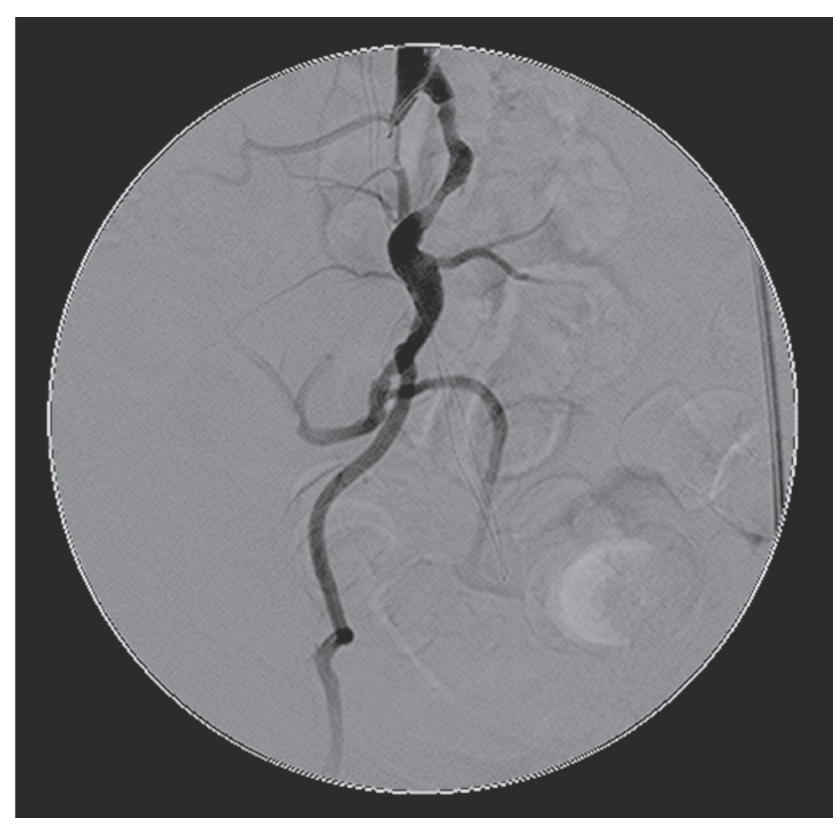

Figura 3. Fio-guia impactando-se na lesão.
Ao exame físico no pós-operatório imediato, evidenciaram-se pulsos femoral, poplíteo, pedioso e tibial posterior em membro inferior direito, ausentes no exame préoperatório. Constatou-se ainda a manutenção dos pulsos do membro inferior esquerdo.

No pós-operatório imediato foi feita dose de ataque de clopidogrel $300 \mathrm{mg}$ e AAS $200 \mathrm{mg}$ ambos, por via oral.

A paciente evoluiu bem no pós-operatório imediato recebendo alta para acompanhamento ambulatorial 24 horas após o procedimento, sem qualquer

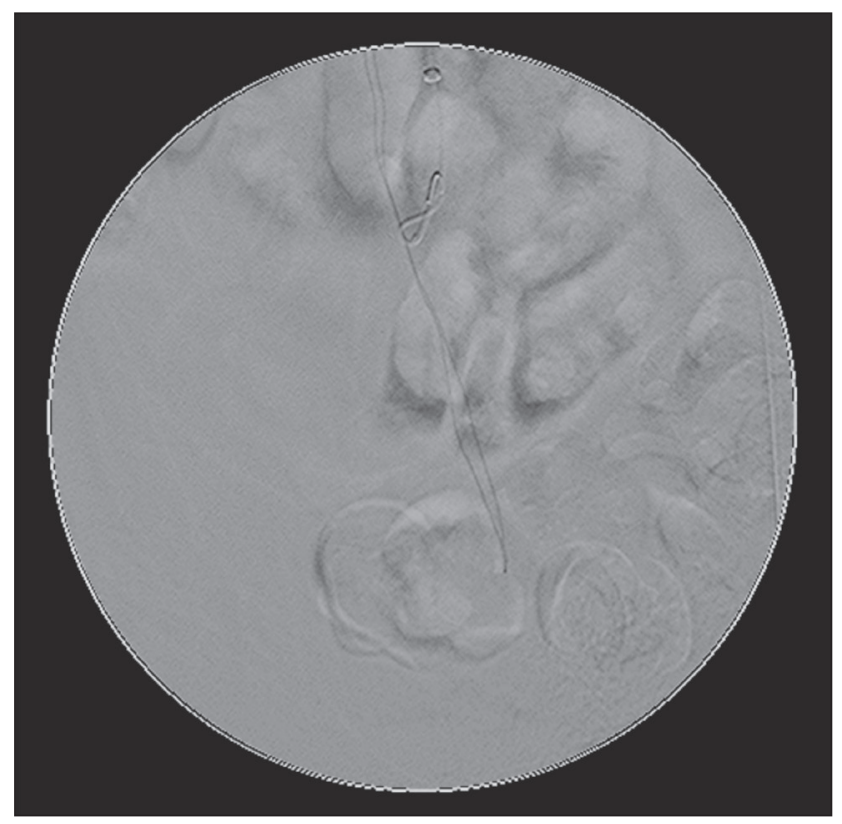

Figura 4. Fio-guia enrolando-se à tentativa de progressão.

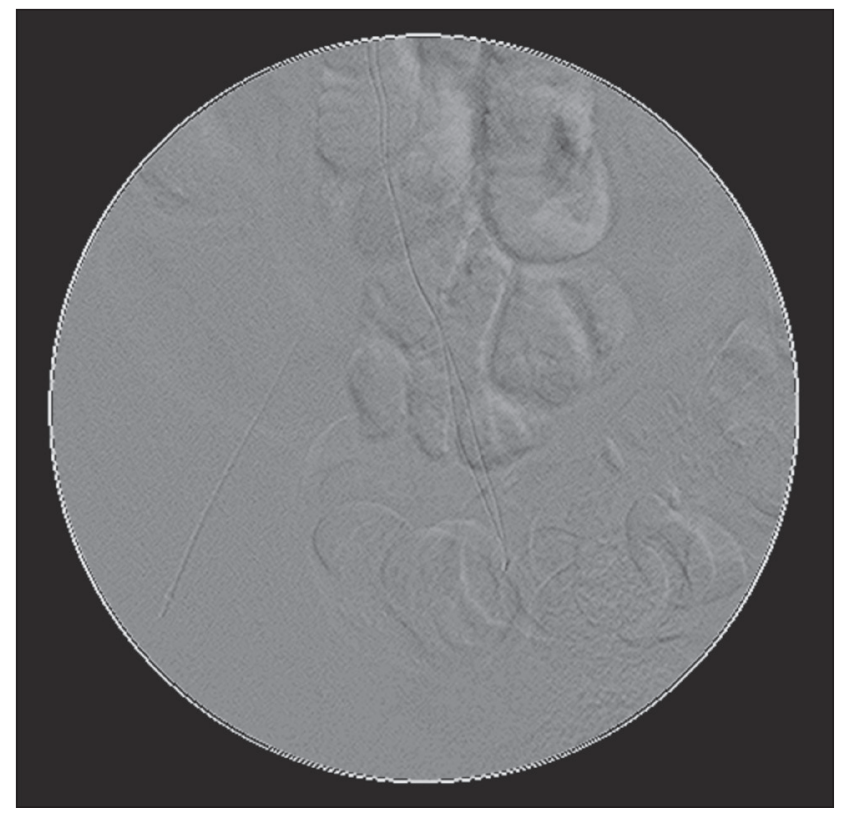

Figura 5. Cateter Frontrunner ${ }^{\circledR}$ transpondo a lesão. 


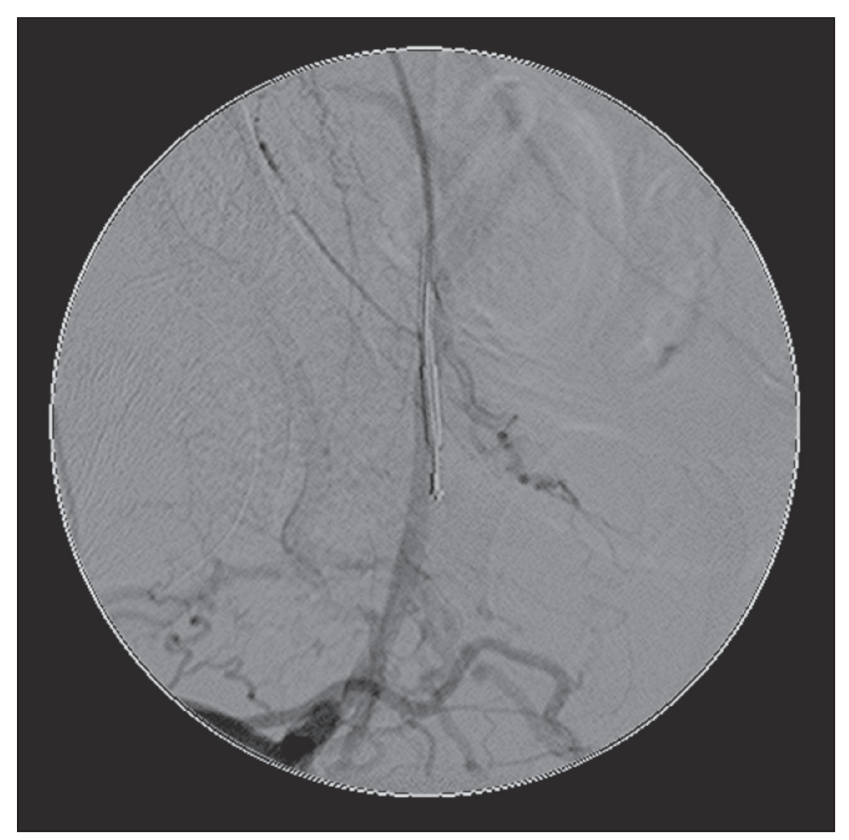

Figura 6. Cateter Outback ${ }^{\circledR}$ subintimal.

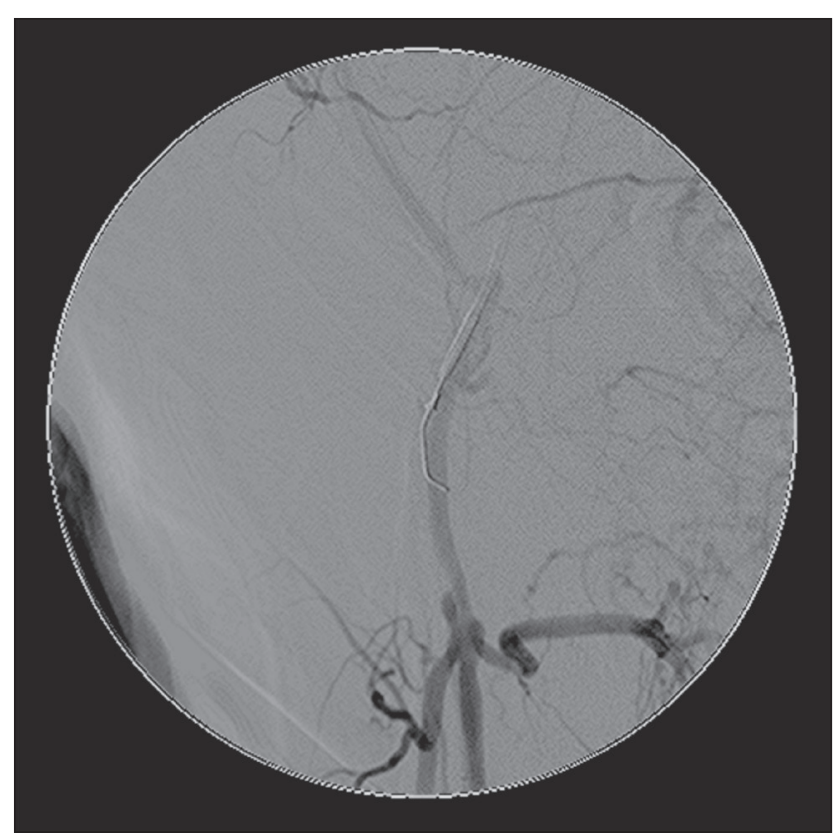

Figura 7. Fio-guia já posicionado na luz arterial.

intercorrência clínica e/ou cirúrgica. Vem em uso regular de clopidogrel $75 \mathrm{mg}$ uma vez ao dia, AAS 100 mg uma vez ao dia e sinvastatina $40 \mathrm{mg}$ uma vez ao dia, além de seus medicamentos de uso habitual para as comorbidades. Em atendimento ambulatorial de retorno, refere remissão completa dos sintomas, execução de caminhada programada diária em torno de 1.000 a 2.000 metros atualmente sem limitação pela claudicação do membro inferior direito.

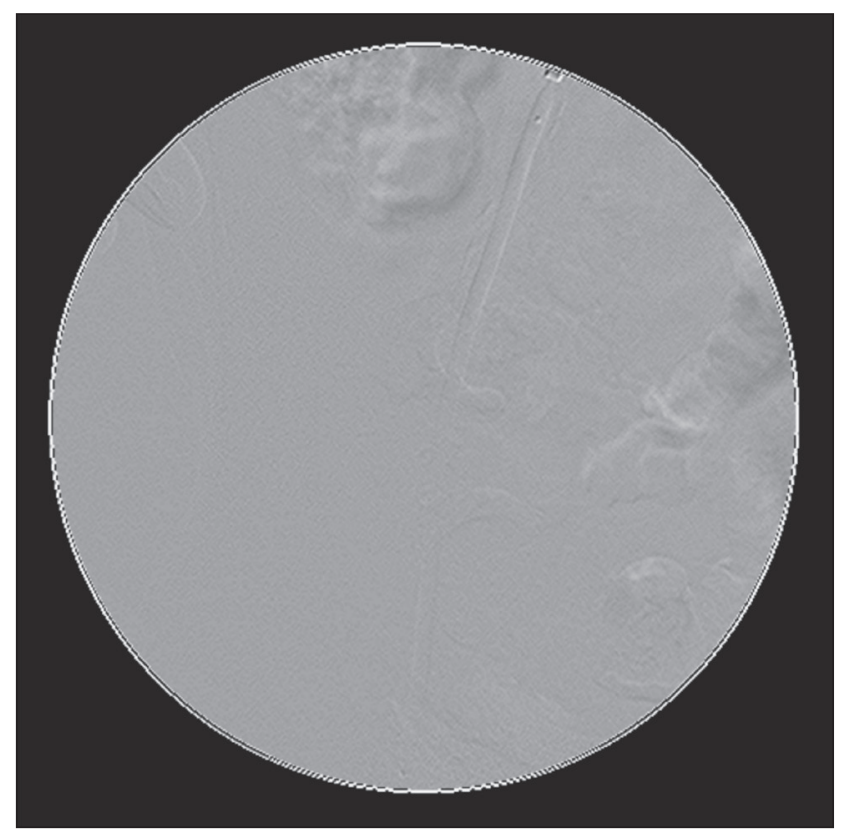

Figura 8. Angioplastia por balão.

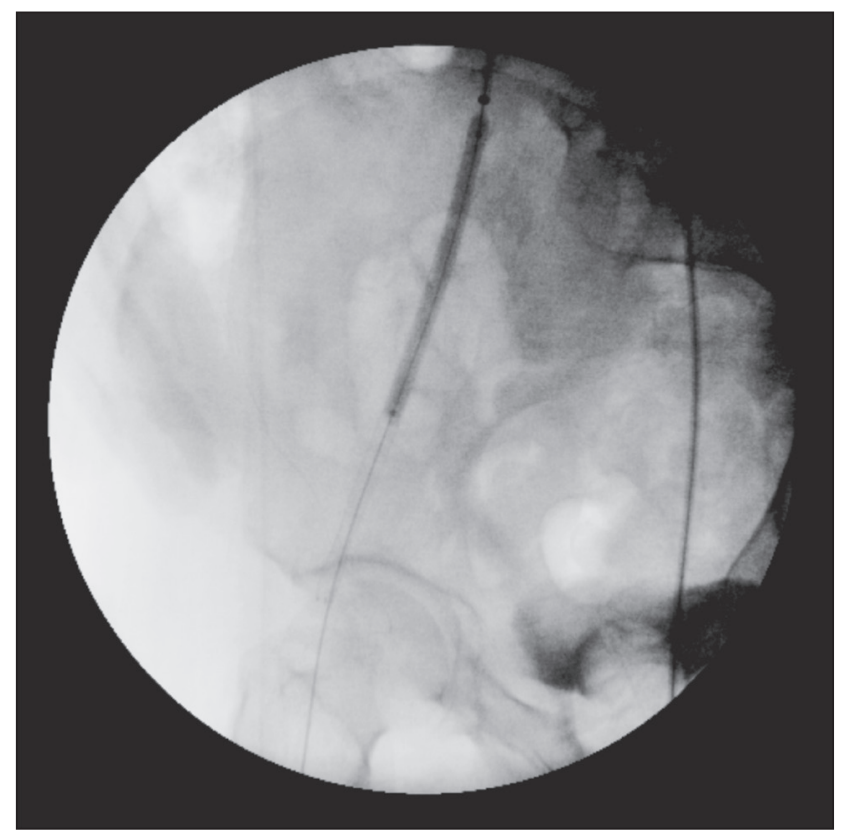

Figura 9. Balonamento após colocação do stent.

\section{Discussão}

A transposição de lesões obstrutivas totais do eixo aorto-ilíaco por técnica endovascular ainda pode ser motivo de dificuldade no tratamento destes pacientes, mesmo nos dias atuais. Este tipo de lesão encontrase presente em 20 a $40 \%$ dos pacientes submetidos ao tratamento endovascular da doença arterial obstrutiva periférica ${ }^{6,7}$. 
Com a evolução contínua no desenvolvimento de novos materiais, há uma tendência de redução dessas dificuldades. No caso acima descrito, o sucesso da terapêutica endovascular não seria possível, não fosse o uso de materiais relativamente novos e que propõem soluções técnicas para problemas específicos que surgem durante a condução do caso. $O$ cateter de transposição de lesão crônica (Frontrunner ${ }^{\circledR}$ ) foi decisivo para o sucesso do procedimento. Trata-se de um cateter com proposta inovadora para transposição de placas calcificadas com taxa de sucesso na ordem de 63 a 76\% ${ }^{8,9}$ em transpor lesões onde o fio-guia não possibilitou a transposição das mesmas. Este cateter possui em sua extremidade distal, um mecanismo articulado que permite a abertura de um pertuito através da placa (Figura 11) ${ }^{10}$. Desta forma possibilita a passagem de um microcateter, dentro do qual navegará um fio-guia 0.014", possibilitando a realização da angioplastia.

Uma adversidade descrita e observada no caso acima relatado foi a saída subintimal do Frontrunner ${ }^{\circledR}$ após a transposição da placa. Assim sendo, novamente a utilização de um material como recurso técnico para situações específicas como essa possibilitou a realização do procedimento, pois a reentrada do guia na luz da artéria pós-lesão só foi possível com a utilização do cateter de reentrada $\left(\right.$ Outback $\left.{ }^{\circledR}\right)$ (Figura 12) ${ }^{10}$, onde um mecanismo de agulha retrátil e direcionável por radioscopia permitiu o retorno do fio-guia para a luz verdadeira. A taxa de sucesso de procedimentos, nos quais foi necessário o uso deste material, gira em torno de $95 \%$ segundo a literatura ${ }^{11}$.

$\mathrm{O}$ uso destes materiais aumenta a taxa de sucesso das revascularizações por via endovascular em pacientes com oclusão arterial total crônica, sem um incremento significativo do risco de acidentes, sendo assim uma alternativa segura para a resolução destes casos onde a progressão simples do fio-guia não é possível.

\section{Conclusão}

É bastante evidente o desenvolvimento na área de bioengenharia assistido na última década. Não obstante, a área da cirurgia endovascular e suas técnicas minimamente invasivas acompanharam tal desenvolvimento visando minimizar suas limitações.

Com relação ao tratamento das lesões obstrutivas crônicas do eixo aorto-ilíaco e ilíaco-femoral, ainda há o que se evoluir no tratamento da patologia aterosclerótica obstrutiva. Porém, o caso relatado exemplifica bem como o avanço tecnológico e o desenvolvimento de novos materiais podem auxiliar de forma significativa, com satisfatória eficiência e segurança, no tratamento de

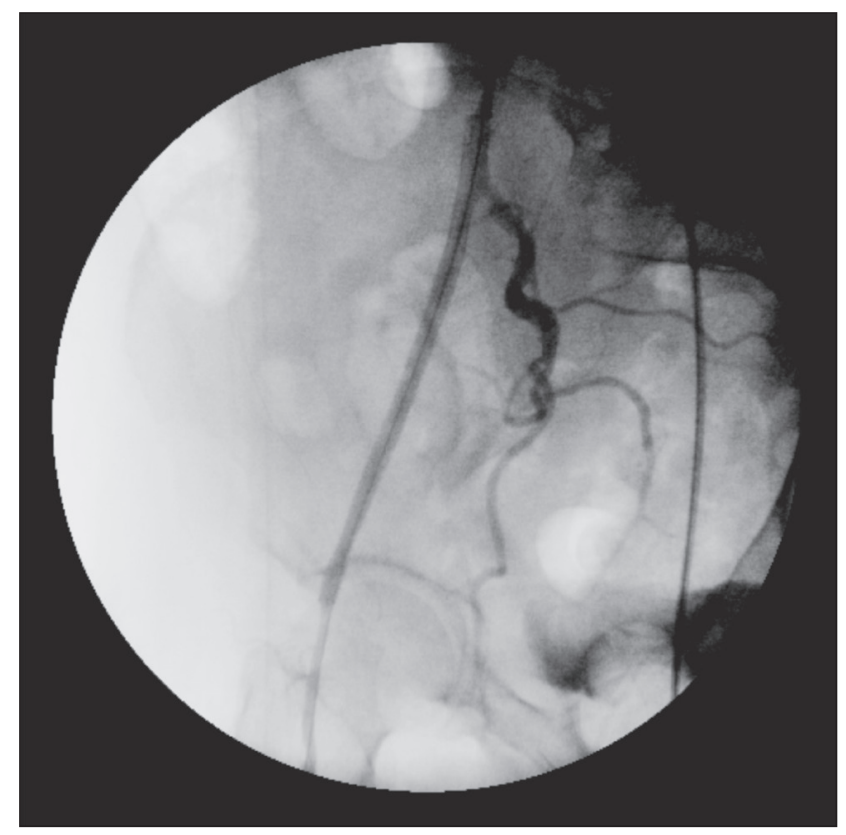

Figura 10. Resultado final.

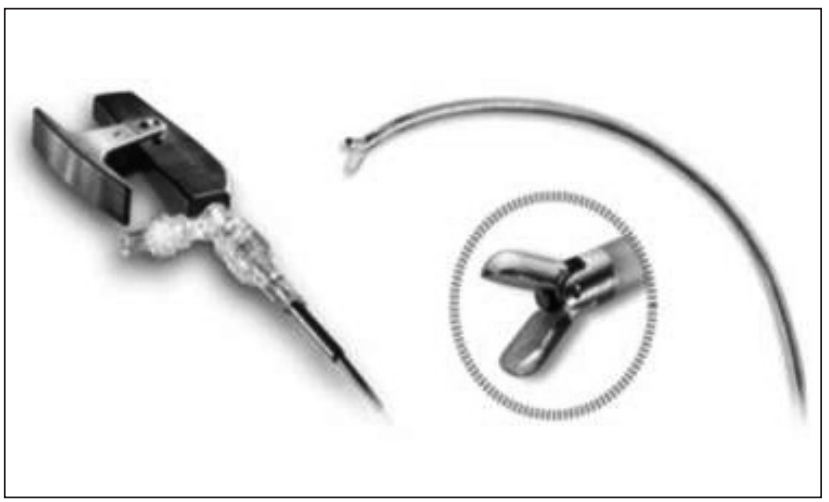

Figura 11. Frontrunner ${ }^{\circledR}$. Fonte: Murarka e Heuser ${ }^{10}$.

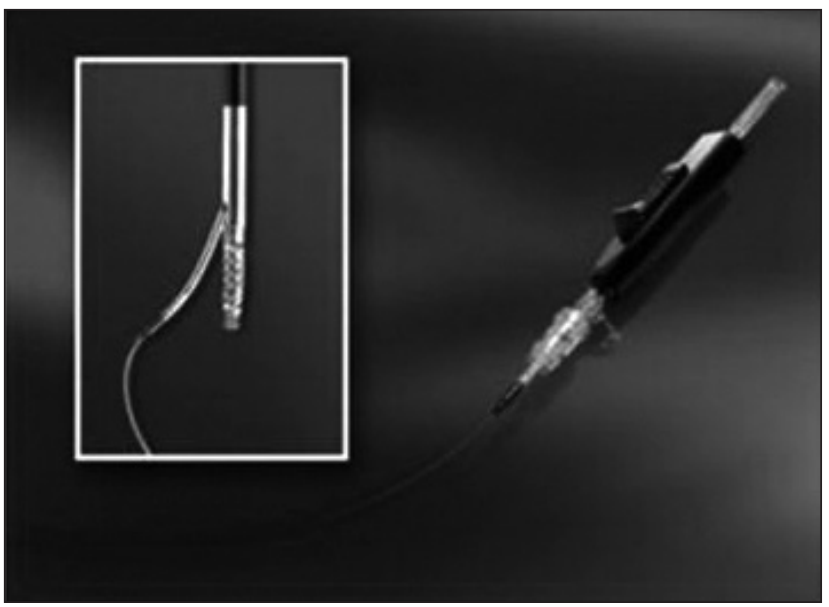

Figura 12. Outback ${ }^{\circledR}$. Fonte: Murarka e Heuser ${ }^{10}$. 
pacientes cuja técnica endovascular apresenta-se como a melhor opção terapêutica cirúrgica.

\section{Agradecimentos}

Agradecemos aos Drs. Rodrigo Andrade Vaz de Melo, Victor Luiz Picão Correa, Luis Cláudio Rosa Arantes e Helen Cristhian Pessoni pela contribuição na condução deste caso.

\section{Referências}

1. Gabriel SA, Serafim PH, Freitas CEM, et al. Doença arterial obstrutiva periférica e índice tornozelo-braço em pacientes submetidos à angiografia coronariana. Rev Bras. Cir Cardiovasc. 2007; 22 (1) 4959. http://dx.doi.org/10.1590/S0102-76382007000100011

2. Nadal LL, Cynamon J, Lipsitz EC. Subintimal angioplasty for chronic arterial occlusions. Tech Vasc Interv Radiol. 2004;7(1):16-22. http://dx.doi.org/10.1590/S0102-76382007000100011

3. Norgren L, Hiatt WR, Dormandy JA, et al. Inter-Society Consensus for the Management of Peripheral Arterial Disease (TASC II). Eur J Vasc Endovasc Surg. 2007;33(Suppl 1):S1-75.

4. Maffei FHA. Doenças vasculares periféricas. 3a.ed. São Paulo: MEDSI, 2002. vol.1 e 2.

5. Rutherford RB, Baker JD, Ernst C, et al. Recommended standards for reports dealing with lower extremity ischemia: revised version. J. Vasc. Surg. 1997; 26(3)517-38. http://dx.doi.org/10.1016/ S0741-5214(97)70045-4

6. Capek P, McLean GK, Berkowitz HD. Femoropopliteal angioplasty: factors influencing long-term success. Circulation. 1991;83(2 Suppl): 17080.
7. Morgenstern BR, Getrajdman GI, Laffey KJ, Bixon R, Martin EC. Total occlusions of the femoropopliteal artery: high technical success rate of conventional balloon angioplasty. Radiology. 1989;172 (3 Pt 2):937-40. PMid:2528164.

8. Melzi G, Cosgrave J, Biondi-Zoccai GL, et al. A novel approach to chronic total occlusions: the crosser system. Catheter Cardiovasc Interv. 2006;68(1):29-35. PMid:16764006.

9. Grube E, Sütsch G, Lim VY, et al. High-frequency mechanical vibration to recanalize chronic total occlusions after failure to cross with conventional guidewires. J Invasive Cardiol. 2006; 18(3):85-91. PMid:16598103.

10. Murarka S, Heuser R. Chronic total occlusions in peripheral vasculature: techniques and devices. Exp Rev Cardiovasc Ther. 2009;7(10):1283-95. PMid:19814671.

11. Joye JD, Serry R, St. Goar FG, Zetterlund P. Clinical success in 100 consecutive endovascular occlusion cases using re-entry catheters for failed sub-intimal approach. J Am Coll Cardiol. 2006; 47 (Suppl 2):31B

Correspondência
Daniel Queiroz Neves
Rua Rodrigues Alves, 100 - Jd. Coolapa
CEP 37950-000 - S. S. Paraíso (MG), Brasil
E-mail: dqneves@yahoo.com.br
Contribuições dos autores
Concepção e desenho do estudo: DQN, JRC), PEOR
Análise e interpretação dos dados: DQN, JRC) GPS, PEOR
Coleta de dados: DQN, JRC), MCTC, MHL, SLA
Redação do artigo: DQN, JRCJ, PEOR
Revisão crítica do texto: PEOR, CLMC
Aprovação final do artigo*: DQN, JRCJ, MCTC, MHL, GPS, CLMC, SLA, PEOR
Análise estatística: N/A
*Todos os autores leram e aprovaram a versão final submetida ao J Vasc Bras.

